PHYSICAL REVIEW D 80, 043528 (2009)

\title{
Stability of the Einstein static universe in the presence of vacuum energy
}

\author{
Saulo Carneiro ${ }^{1,2, *}$ and Reza Tavakol ${ }^{1, \dagger}$ \\ ${ }^{1}$ Astronomy Unit, School of Mathematical Sciences, Queen Mary University of London, Mile End Road, London E1 4NS, \\ United Kingdom \\ ${ }^{2}$ Instituto de Física, Universidade Federal da Bahia, Salvador, BA, 40210-340, Brazil
}

(Received 26 June 2009; published 25 August 2009)

\begin{abstract}
The Einstein static universe has played a central role in a number of emergent scenarios recently put forward to deal with the singular origin of the standard cosmological model. Here we study the existence and stability of the Einstein static solution in the presence of vacuum energy corresponding to conformally invariant fields. We show that the presence of vacuum energy stabilizes this solution by changing it to a center equilibrium point, which is cyclically stable. This allows nonsingular emergent cosmological models to be constructed in which initially the Universe oscillates indefinitely about an initial Einstein static solution and is thus past eternal.
\end{abstract}

DOI: 10.1103/PhysRevD.80.043528

PACS numbers: $98.80 . \mathrm{Cq}$

\section{INTRODUCTION}

Recent accumulation of high resolution observations is compatible with the so-called standard model of cosmology, which has a number of intriguing features. In addition to the early and late accelerating phases, which are difficult to account for within the classical relativistic framework with nonexotic matter sources, this model also possesses an initial singular state at which the laws of physics break down. To deal with this latter shortcoming, a number of attempts have recently been made to construct models which are nonsingular and/or past eternal. ${ }^{1}$ These fall into a number of groups, including emergent scenarios [3-5] (see also [6,7]) and cyclic/ekpyriotic models [8]. There are also a number of other cosmological models which are not necessarily recurrent, but nevertheless are nonsingular in the past. These include the pioneering nonsingular model by Bojowald based on modifications due to loop quantum gravity [9], the model based on polymer matter [10], and others [11].

The so-called emergent scenarios, which are nonsingular and past eternal, come in two varieties: those that employ classical general relativity $[3,4]$, and those that incorporate quantum effects which are expected to be present at early phases of the Universe [5]. An important ingredient in the construction of these models is the Einstein static solution, which in the classical general relativistic setting has long been known to be unstable. ${ }^{2}$ This instability makes the construction of emergent models within the framework of classical relativity difficult.

\footnotetext{
*ICTP Associate Member; saulo.carneiro@pq.cnpq.br

r.tavakol@qmul.ac.uk

${ }^{1}$ The history of attempts at constructing nonsingular/oscillatory universes which are past eternal goes much further back to at least the work of Tolman [1] (see also [2] and references therein for a review of nonsingular models).

${ }^{2}$ This solution has, however, been shown to be stable with respect to inhomogeneous perturbations [12]).
}

Interestingly though, quantum effects which are known to be operative at early phases of the Universe have recently been shown to be able to stabilize the Einstein static solution by changing it from a hyperbolic equilibrium point (which is unstable) to a center equilibrium point, which is cyclically stable. This was first shown to be the case in presence of quantum modifications due to loop quantumgravity effects in [5] (see also $[13,14]$ and other related works in this connection $[15,16])$. In most cases, where the singularity is removed, the overall effect is to change the effective evolution equations in such a way which allows the singularity theorems to be circumvented. For example, in the context of loop quantum cosmology it has been shown (see [5] for details) that, by writing the effective evolution equations in terms of an effective fluid, the equation of state in the semiclassical regime can change, and even become phantom (with equation-of-state parameter $<-1$ ). This results in the violation of the null energy condition which removes a crucial barrier to singularity avoidance.

Here we consider an ingredient not studied before, namely, the contribution of vacuum quantum fluctuations to the energy content of classical Einstein equations, and study their effect on the existence and stability of the Einstein static solution. In this connection we recall an earlier result [17], according to which the vacuum energy of conformal fields in a spatially closed static universe has a density proportional to $a^{-4}$, where $a$ is the scale factor. This result has been confirmed more recently by other studies which show that the Casimir energy in such backgrounds is proportional to $a^{-1}$ [18]. We study the effects of presence of this vacuum energy on the dynamics of the Universe and show that it has the consequence of stabilizing the Einstein static solution by changing it into a center equilibrium point.

The outline of the paper is as follows. In Sec. II we perform a general analysis of the equilibrium of Einstein solution in the presence of matter and the vacuum energy. 
In Sec. III we consider a numerical example, in which the energy content is assumed to consist of relativistic matter plus a vacuum term with negative pressure. In Sec. IV we study the existence of an Einstein static solution in the presence of a single scalar field, with the vacuum contribution represented by a self-interaction potential. We then briefly discuss possible alternatives for the exit from the initial Einstein phase to an inflationary one. We conclude with a discussion in Sec. V.

\section{STUDY OF THE EINSTEIN UNIVERSE IN THE GENERAL SETTING}

In this section we study the effects of vacuum energy due to conformal fields on the dynamics of the Universe, and, in particular, the way this affects the existence and the stability of the Einstein static solution. As was mentioned above, the vacuum energy of a conformal scalar field in a spatially closed static universe of radius $a$ has been shown to have a density given by $\rho_{\Lambda}=C / a^{4}$, where $C$ is a positive constant [17]. Initially it was also proposed that the corresponding pressure should have the form $p_{\Lambda}=$ $\rho_{\Lambda} / 3$, to ensure the vacuum energy-momentum tensor is traceless in order to respect the conformal symmetry. However, with the discovery of a trace anomaly such a requirement is not necessary. In the de Sitter space-time the equation-of-state parameter of the vacuum is $\omega_{\Lambda}=-1$, due to the symmetry of the background. This result, however, cannot be assumed to hold in other more general space-times. Here, therefore, we shall proceed by first considering the general case, $p_{\Lambda}=\omega_{\Lambda} \rho_{\Lambda}$, where $\omega_{\Lambda}$ is allowed to take arbitrary values. We shall then find the conditions for the stability of the Einstein static universe in terms of $\omega_{\Lambda}$.

Starting with a closed isotropic and homogeneous Friedmann-Lemaître-Robertson-Walker (FLRW) model sourced by a general fluid with total density $\rho$ and total pressure $p$, the evolution equations are given by

$$
\begin{gathered}
3 H^{2}=\rho-\frac{3}{a^{2}}, \\
\dot{\rho}+3 H(\rho+p)=0,
\end{gathered}
$$

where $H$ is the Hubble parameter. We shall assume the fluid to consist of a combination of the above vacuum energy plus matter with $p_{m}=\omega_{m} \rho_{m}$, where $\rho_{m}, p_{m}$, and $\omega_{m}$ are the corresponding density, pressure, and equationof-state parameter. Substituting these in the above evolution equations and letting $\rho_{\Lambda}=C / a^{4}$, the Raychadhuri equation can be written as

$$
\ddot{a}=-\frac{\dot{a}^{2}+1}{2 a}\left(1+3 \omega_{m}\right)+\frac{C}{2 a^{3}}\left(\omega_{m}-\omega_{\Lambda}\right) .
$$

The Einstein static solution is given by $\ddot{a}=0=\dot{a}$. To begin with we obtain the conditions for the existence of this solution. The scale factor in this case is given by

$$
a_{\mathrm{ES}}^{2}=\frac{C\left(\omega_{m}-\omega_{\Lambda}\right)}{3 \omega_{m}+1} .
$$

The existence condition reduces to the reality condition for $a_{\mathrm{ES}}$, which for a positive $C$ takes the forms

$$
\omega_{m}>-1 / 3 \text { and } \omega_{\Lambda}<\omega_{m}
$$

or

$$
\omega_{m}<-1 / 3 \text { and } \omega_{\Lambda}>\omega_{m} .
$$

Therefore, in the case of ordinary matter $\left(\omega_{m} \geq 0\right)$ plus a positive vacuum energy with negative pressure (i.e., with $\left.\omega_{\Lambda}<0\right)$, the Einstein static solution always exists. ${ }^{3}$

To study the stability of this solution, it is helpful to cast Eq. (3) as a two-dimensional dynamical system by introducing the phase-space variables $x_{1}=a$ and $x_{2}=\dot{a}$,

$$
\begin{gathered}
\dot{x}_{1}=x_{2}, \\
\dot{x}_{2}=-\frac{x_{2}^{2}+1}{2 x_{1}}\left(1+3 \omega_{m}\right)+\frac{C}{2 x_{1}^{3}}\left(\omega_{m}-\omega_{\Lambda}\right) .
\end{gathered}
$$

In these variables the Einstein static solution corresponds to the fixed point $\left(x_{1}=a_{\mathrm{ES}}, x_{2}=0\right)$. The stability of this equilibrium point is readily found by looking at the eigenvalues, $\lambda$, of the Jacobian matrix $J_{i j}=\partial \dot{x}_{i} / \partial x_{j}$ evaluated at this point, which are found to be

$$
\lambda^{2}=-\frac{C\left(\omega_{m}-\omega_{\Lambda}\right)}{a_{\mathrm{ES}}^{4}} .
$$

The stability depends on the sign of $\lambda^{2}$. For $\lambda^{2}>0$, the Einstein static solution is a hyperbolic fixed point and hence unstable, in the sense that trajectories starting in the neighborhood of such a point exponentially diverge from it (this is the same as the classical relativistic case). For $\lambda^{2}<0$, on the other hand, the Einstein static solution becomes a center equilibrium point, which is circularly stable, in the sense that small departures from the fixed point result in oscillations about that point rather than exponential deviation from it. In this case the Universe stays (oscillates) in the neighborhood of the Einstein static solution indefinitely. Thus the condition for stability is given by $\lambda^{2}<0$. For $C>0$, this implies that $\omega_{\Lambda}<\omega_{m}$. Comparing this inequality with the conditions for existence of the Einstein static solution, (5) and (6), we conclude that, given $\omega_{\Lambda}<\omega_{m}$, the Einstein universe is stable for

\footnotetext{
${ }^{3}$ It is interesting to note that conditions (5) and (6) exclude the case $\omega_{\Lambda}=1 / 3$, originally proposed in [17].
} 

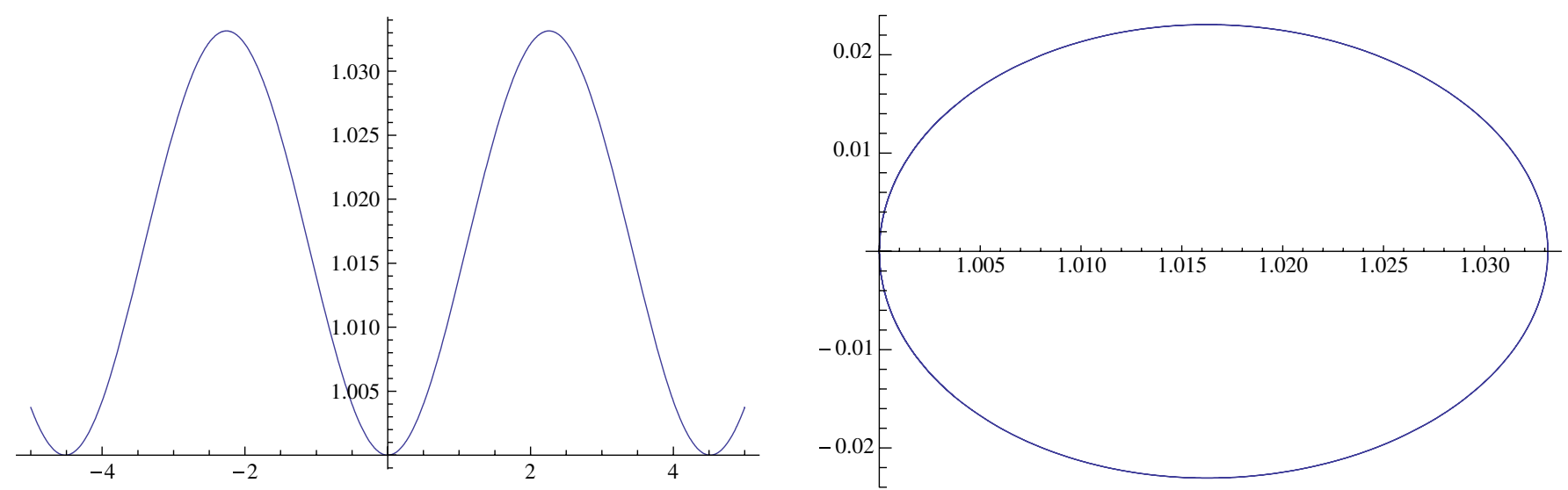

FIG. 1 (color online). The scale factor as a function of time (left) and a typical trajectory in the phase space $(a, \dot{a})$ (right).

$\omega_{m}>-1 / 3$. In particular, it is stable in the presence of ordinary matter $\left(\omega_{m} \geq 0\right)$ plus a positive vacuum energy with negative pressure. ${ }^{4}$

To close this section, we note that perturbations about the fixed point imply perturbations in $a$ which in turn imply perturbations in $\rho_{\Lambda}$. In the case of vacuum energy with $\omega_{\Lambda}=-1$, this implies a coupling between matter and vacuum. Since the conservation of total energy is guaranteed by (2), the quantum vacuum would have to exchange energy with matter. We shall consider this coupling in Sec. IV below, using an alternative approach. We should emphasize here that this coupling is essential for the above results to hold. This may explain why, when matter and vacuum are independently conserved and $\omega_{\Lambda}=-1$, the stability is achieved only for $\omega_{m}<-1 / 3[8,15]$. In the next section we shall explicitly demonstrate this coupling with the help of a numerical example.

\section{NUMERICAL STUDY OF THE COSMOLOGICAL DYNAMICS}

In this section we make a brief quantitative study of the effects of the vacuum energy on the dynamics of the Universe. As an example, we consider the case where the energy content consists of vacuum energy, which we assume to have $\omega_{\Lambda}=-1$ and $\rho_{\Lambda}=C / a^{4}$, plus a relativistic matter with an equation-of-state parameter $\omega_{\text {rm }}=1 / 3$. Using these equation-of-state parameters in Eq. (3) we obtain

$$
3 a^{3} \ddot{a}+3 a^{2} \dot{a}^{2}+3 a^{2}-2 C=0 .
$$

For the Einstein static solution the corresponding scale factor is given by $a^{2}=2 C / 3$. Clearly phase-space trajectories starting exactly on the Einstein static fixed point

\footnotetext{
${ }^{4}$ In the case of a negative vacuum energy, that is $C<0$, the conditions for existence and stability of the Einstein solution are given by $\omega_{\Lambda}>\omega_{m}>-1 / 3$, which are satisfied by any ordinary matter, provided the negative vacuum term has negative pressure.
}

would remain there indefinitely. On the other hand, trajectories starting in the neighborhood of this point would oscillate indefinitely about this solution. In Fig. 1 we have plotted an example of such a universe trajectory using initial conditions given by $a(0)=1$ and $\dot{a}(0)=0$, together with $2 C=3.1$.

To explicitly demonstrate the coupling between the matter and vacuum components, discussed above, we also plot in Fig. 2 the time dependence of the energy densities of these components. In the absence of coupling, we would have a constant $\rho_{\Lambda}$ and a constant $\rho_{\text {rm }} a^{4}$ for relativistic matter. In our case, however, the effect of the coupling is to make these quantities oscillate with time, as can be seen from Fig. 2.

\section{THE SCALAR FIELD ANALOGY}

As discussed above, a perturbed Einstein universe with $\rho_{\Lambda}=C / a^{4}$ and $\omega_{\Lambda}=-1$ is only possible if the quantum vacuum exchanges energy with matter. In this section we consider an alternative procedure, originally introduced in the case of de Sitter space-time [7], in which such a coupling is modeled in terms of a minimally coupled scalar field $\phi$ with a self-interaction potential $V$. This can be done by interpreting the usual expressions for the energy density and the pressure of the scalar field,

$$
\rho_{\phi}=\dot{\phi}^{2} / 2+V
$$

$$
p_{\phi}=\dot{\phi}^{2} / 2-V
$$

as a sum of a vacuum energy component with density $V$ and pressure $-V$, plus a stiff fluid component with density and pressure equal to $\dot{\phi}^{2} / 2$. Now, when the scale factor is perturbed, there is an energy exchange between the potential energy $V$ (here representing the vacuum and taken to be $V=C / a^{4}$ ) and the kinetic energy $\dot{\phi}^{2} / 2$. 

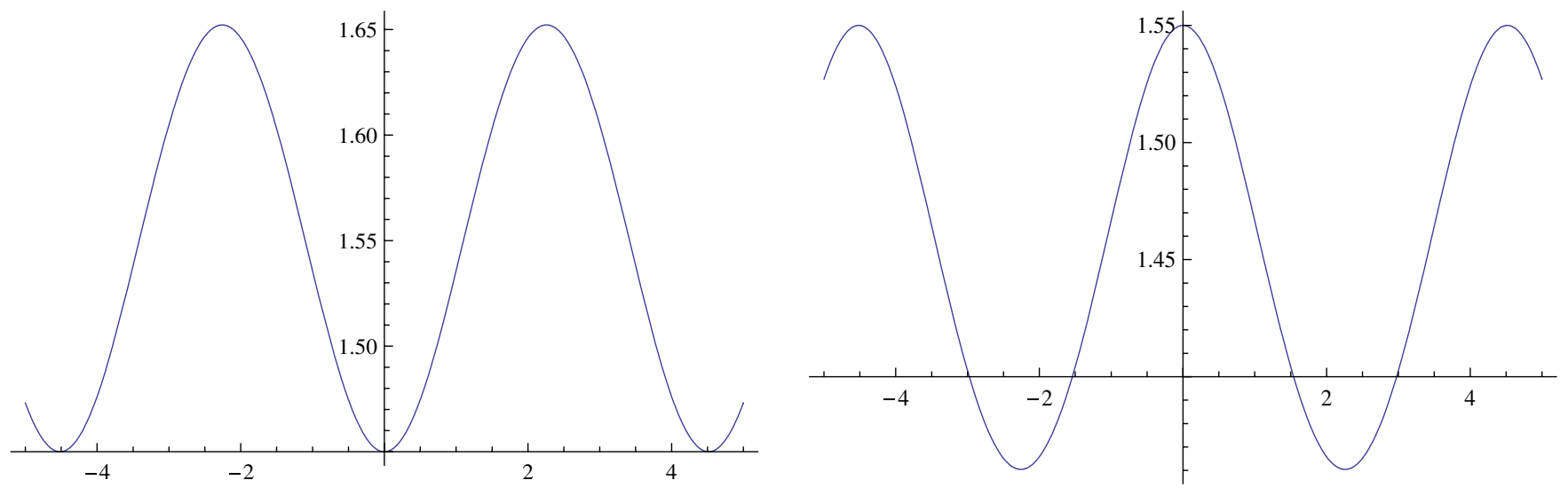

FIG. 2 (color online). The time dependence of $\rho_{\mathrm{rm}} a^{4}$ (left) and $\rho_{\Lambda}$ (right).

The Lagrangian in this case takes the usual form

$$
\mathcal{L}=\sqrt{-g}\left[\frac{R}{2}-\frac{1}{2}(\partial \phi)^{2}-V(\phi)\right] .
$$

For an FLRW universe with positive curvature, the evolution equations take their usual forms:

$$
\begin{gathered}
3 H^{2}=V+\frac{\dot{\phi}^{2}}{2}-\frac{3}{a^{2}}, \\
\frac{\dot{\phi}^{2}}{2}=-\dot{H}+\frac{1}{a^{2}} .
\end{gathered}
$$

Using (11) and (12), it is not difficult to cast the above system in the form of the evolution Eqs. (1) and (2). Taking for the potential the expression $V=C / a^{4}$, we can derive the Raychaudhuri equation in the form

$$
a^{3} \ddot{a}+2 a^{2} \dot{a}^{2}+2 a^{2}-C=0 .
$$

The behavior of this system can be readily studied using the general analysis given in Sec. II, by recalling that in this case we have a mixture of a vacuum term (with $\omega_{\Lambda}=-1$ ) plus a stiff fluid (with $\omega_{m}=1$ ). The Einstein static solution again corresponds to $\ddot{a}=0=\dot{a}$, which in this case gives the corresponding scale factor to be $a^{2}=C / 2$. A similar analysis to that used above shows that this fixed point is again a center.

It is also instructive to briefly revisit the original classical emergent model proposed in [3] in terms of this alternative formulation of the dynamics in terms of a scalar field. In that scenario also, the initial Einstein static phase has a scalar field as its energy content. We can see from (14) and (15) that, for the Einstein static solution, $\dot{\phi}^{2}$ and $V$ are both nonzero constants. Therefore, while $\phi$ is changing with time, $V$ is not. In other words, the scalar field is rolling along a potential plateau. As discussed in [3], this plateau may be considered as the past-asymptotic limit of a smoothly decreasing potential, which eventually leads to an exit from the Einstein static regime into an inflationary phase. Specific forms of such a potential have been considered in [3-5].

Finally, another possibility that may be considered is that of a complex scalar field. For example, with a harmonic field $\phi=\phi_{0} e^{i \omega t}$ we have $\dot{\phi}^{2}=\omega^{2} \phi_{0}^{2}$, and Eqs. (14) and (15) are simultaneously satisfied, with $V=$ $\omega^{2} \phi_{0}^{2}$. Therefore, $V$ remains constant while $\phi$ rotates in the complex plane. The stability of the solution indicates that this is a local minimum of $V$, and the exit to the inflationary phase may involve, for example, a tunnelling to a global minimum.

\section{CONCLUSION}

We have studied the existence and stability of the Einstein static universe in the presence of vacuum energy corresponding to conformally invariant fields. Using the result that the vacuum energy density in Einstein universe is proportional to the inverse fourth power of the scale factor, we have found the range of equation-of-state parameters for the vacuum energy such that the Einstein universe is stable, in the sense of dynamically corresponding to a center equilibrium point. The importance of such a solution is due to the central role it plays in the construction of nonsingular emergent oscillatory models which are past eternal, and hence can resolve the singularity problem in the standard cosmological scenario.

Given that the oscillatory universe discussed above is close to but not exactly an Einstein universe, the form of the vacuum energy density in the initial oscillatory phase of the Universe may depart from the above inverse fourthpower form. To partially answer what happens if vacuum density takes other forms, we considered, as a first step in this direction, a more general functional form of the type

$$
\rho_{\Lambda}=C / a^{n} .
$$

Proceeding in a similar manner to that used above we have been able to show that, in the case where the content of the Universe consists of radiation (with $\omega_{m}=1 / 3$ ) plus vac- 
uum energy (with $\omega_{\Lambda}=-1$ ), the Einstein static universe still exists and is stable (is a center fixed point) if $n>2$.

\section{ACKNOWLEDGMENTS}

Saulo Carneiro was partially supported by CAPES (Brazil).
[1] R. C. Tolman, Relativity, Thermodynamics and Cosmology (Clarendon Press, Oxford, 1934).

[2] M. Novello and S.E. Perez Bergliaffa, Phys. Rep. 463, 127 (2008).

[3] G. F. R. Ellis and R. Maartens, Classical Quantum Gravity 21, 223 (2004).

[4] G.F. R. Ellis, J. Murugan, and C. G. Tsagas, Classical Quantum Gravity 21, 233 (2004).

[5] D. J. Mulryne, R. Tavakol, J. E. Lidsey, and G. F. R. Ellis, Phys. Rev. D 71, 123512 (2005).

[6] S. Carneiro, Phys. Rev. D 61, 083506 (2000).

[7] S. Carneiro and R. Tavakol, arXiv:0905.3131 [Gen. Relativ. Gravit. (to be published)].

[8] J. Khoury, B. A. Ovrut, P. J. Steinhardt, and N. Turok, Phys. Rev. D 64, 123522 (2001); P. J. Steinhardt and N. Turok, Science 296, 1436 (2002); Phys. Rev. D 65, 126003 (2002); J. Khoury, P. J. Steinhardt, and N. Turok, Phys. Rev. Lett. 92, 031302 (2004); Y. Shtanov and V. Sahni, Phys. Lett. B 557, 1 (2003); J. D. Barrow, D. Kimberly, and J. Magueijo, Classical Quantum Gravity 21, 4289 (2004); J. D. Barrow and C. G. Tsagas, arXiv:0904.1340.

[9] M. Bojowald, Phys. Rev. Lett. 86, 5227 (2001).

[10] G. M. Hossain, V. Husain, and S.S. Seahra, arXiv:
0906.2798.

[11] V. Sahni and L. A. Kofman, Phys. Lett. A 117, 275 (1986); R. Ferraro and F. Fiorini, Phys. Rev. D 78, 124019 (2008).

[12] J. D. Barrow, G. F. R. Ellis, R. Maartens, and C. Tsagas, Classical Quantum Gravity 20, L155 (2003).

[13] D. J. Mulryne, N. J. Nunes, R. Tavakol, and J. E. Lidsey, Int. J. Mod. Phys. A 20, 2347 (2005).

[14] G. V. Vereshchaign, J. Cosmol. Astropart. Phys. 07 (2004) 013.

[15] C. G. Boehmer, L. Hollenstein, and F. S. N. Lobo, Phys. Rev. D 76, 084005 (2007); R. Goswami, N. Goheer, and P. K. S. Dunsby, Phys. Rev. D 78, 044011 (2008); U. Debnath, Classical Quantum Gravity 25, 205019 (2008); S. S. Seahra and C. G. Boehmer, Phys. Rev. D 79, 064009 (2009); C. G. Boehmer and F. S. N. Lobo, Phys. Rev. D 79, 067504 (2009).

[16] L. Parisi, M. Bruni, R. Maartens, and K. Vandersloot, Classical Quantum Gravity 24, 6243 (2007).

[17] L. H. Ford, Phys. Rev. D 11, 3370 (1975); 14, 3304 (1976).

[18] E. Elizalde and A.C. Tort, Mod. Phys. Lett. A 19, 111 (2004); I. H. Brevik, K. A. Milton, and S.D. Odintsov, arXiv:hep-th/0210286. 